\title{
Preparation of Bioactive Titanium Surfaces via Fluoride and Fibronectin Retention
}

\author{
Carlos Nelson Elias, ${ }^{1}$ Patricia Abdo Gravina, ${ }^{1}$ \\ Costa e Silva Filho, ${ }^{2}$ and Pedro Augusto de Paula Nascente ${ }^{3}$ \\ ${ }^{1}$ Biomaterials Laboratory, Instituto Militar de Engenharia, Pr Gen Tibúrcio 80, \\ 22290-270 Rio de Janeiro, RJ, Brazil \\ ${ }^{2}$ Avenue Carlos Chagas Filho, 373, Cidade Universitária-21941-902 Rio de janeiro, RJ, Brazil \\ ${ }^{3}$ Rodovia Washington Luís km 235, 13565-905 São Carlos, SP, Brazil \\ Correspondence should be addressed to Carlos Nelson Elias, elias@ime.eb.br
}

Received 4 August 2012; Accepted 26 September 2012

Academic Editor: Paulo Guilherme Coelho

Copyright () 2012 Carlos Nelson Elias et al. This is an open access article distributed under the Creative Commons Attribution License, which permits unrestricted use, distribution, and reproduction in any medium, provided the original work is properly cited.

\begin{abstract}
Statement of Problem. The chemical or topographic modification of the dental implant surface can affect bone healing, promote accelerated osteogenesis, and increase bone-implant contact and bonding strength. Objective. In this work, the effects of dental implant surface treatment and fibronectin adsorption on the adhesion of osteoblasts were analyzed. Materials and Methods. Two titanium dental implants (Porous-acid etching and PorousNano-acid etching followed by fluoride ion modification) were characterized by high-resolution scanning electron microscopy, atomic force microscopy, and X-ray diffraction before and after the incorporation of human plasma fibronectin (FN). The objective was to investigate the biofunctionalization of these surfaces and examine their effects on the interaction with osteoblastic cells. Results. The evaluation techniques used showed that the Porous and PorousNano implants have similar microstructural characteristics. Spectrophotometry demonstrated similar levels of fibronectin adsorption on both surfaces (80\%). The association indexes of osteoblastic cells in FN-treated samples were significantly higher than those in samples without FN. The radioactivity values associated with the same samples, expressed as counts per minute $(\mathrm{cpm})$, suggested that FN incorporation is an important determinant of the in vitro cytocompatibility of the surfaces. Conclusion. The preparation of bioactive titanium surfaces via fluoride and FN retention proved to be a useful treatment to optimize and to accelerate the osseointegration process for dental implants.
\end{abstract}

\section{Introduction}

The phenomenon of endosseous implant osseointegration, conceptualized by Branemark as the "direct, structural and functional link between the living and orderly bone and the surface of an implant subjected to functional loads" [1], is fundamental to the success of dental implant applications. Commercially pure titanium (cp Ti) is the main material used for this purpose because it has good biocompatibility and adequate mechanical strength. Ti exposed to oxidizing agents spontaneously forms a 10-100 $\AA$ thick titanium oxide layer. This layer is stable in most media, especially under physiological conditions, and, surgically, it shows no change in thickness or corrosion. This ensures implant-bone tissue interaction and osseointegration [2]. The reactions of the tissue host with the biomaterial are determined by the surface properties of the biomaterial. The dental implant surface treatment should induce the differentiation of the desired cells [3]. Surface treatments of available implants promote changes in the mechanical, microstructural, and physical properties, as well as the wettability, energy, chemical composition, and density of chemical groups or molecules on the surface $[2,4]$.

This paper shows that the bone-implant interface strength is greater in dental implants with rough surfaces than in those with smooth surfaces $[5,6]$. Treatments to increase the surface area for fibrin adhesion encourages implant adhesion. The presence of these surfaces also 
increases platelet activation, which produces large gradients of cytokines and growth factors through which leukocytes and osteogenic cells can penetrate the healing site [7]. Titanium surfaces coated with proteins can influence host reactions and thus enhance tissue integration [4]. Fibronectin is a major adhesion protein in the extracellular membrane, and it is important for cell adhesion, migration, proliferation, differentiation, and survival because it facilitates focal contacts with the receptors.

Appropriate changes in dental implant surface roughness can produce better anchoring strength and mechanical locking in the early stages of osseointegration $[2,6]$. Moreover, surfaces with different microtopographies provide a larger area for fibrin adhesion, potentiate platelet activation, and favorably affect local angiogenesis and cellular functions including migration, alignment, orientation, attachment, and differentiation $[5,6]$.

Johansson et al. [8] observed that surfaces treated with fluoride are smoother than sandblasted surfaces but that fluoride-treated surfaces showed higher calcium-phosphorus binding capacity, which could indicate an increased ability of the surface to react with calcified tissues and promote integration between bone and implant. According to Ellingsen and Lyngstadaas [9], in vitro tests have shown that titanium fluoride treatments have a greater capacity for the nucleation of phosphate crystals than sandblasted $\mathrm{Ti}$ implants. In vivo fluoride ion-modified implants have generally proven superior to sandblasted surfaces in terms of osseointegration, ultimately increasing the removal torques.

Fibronectin is a major extracellular matrix protein that is known to promote cell attachment and spreading, differentiation, and phagocytosis. It is a dimeric glycoprotein found in all vertebrates in two basic forms: soluble (plasma and other fluids) and insoluble (extracellular matrix of various tissues). It has a molecular weight between 440 and $500 \mathrm{kDa}$. Disulfide bridges link one subunit to another via sites near the carboxy termini of each subunit. The fibronectin protein has folds that lead to structural remodeling and various conformations according to the medium [10-12].

Fibronectin (FN) functions in cell adhesion, migration, survival, proliferation, and differentiation as well as tissue organization. The FN molecule can interact with other biomolecules, such as collagen, proteoglycan, heparin, hyaluronic acid, fibrin/fibrinogen, plasmin, gangliosides, complement components, and also integral proteins of cell plasma membrane-integrins, as well as with itself [13].

Menezes [10] conducted a study to assess the interaction of human osteoblasts with films of human plasma fibronectin prepared under different $\mathrm{pH}$ conditions. The results showed no quantitative differences in the interaction of human osteoblastic cells (HOB) to different coatings, but qualitative differences were observed; osteoblasts adhered to each of the substrates in very different ways. The largest areas of cells adhesion were observed for substrates preincubated at $4.5 \mathrm{pH}$.

Petrie et al. [14] conducted a clinical study to evaluate the effects of specific bioactive coatings on the healing of bone tissue and the osseointegration of titanium dental implants. The author showed that surfaces containing a
FN fragment for the integrin $\alpha 5 \beta 1$ (FNIII $^{7-10}$ ) increase osteoblastic differentiation and optimize tissue formation and functional integration compared with untreated surfaces or surfaces containing only the RGD sequence.

The purpose of this study was to evaluate the effect of the fluoride treatment of cp titanium samples on the adhesion and proliferation of osteoblastic cells on surfaces with and without fibronectin coating.

\section{Materials and Methods}

2.1. Samples. Implants and discs of grade 4 machined $\mathrm{cp} \mathrm{Ti}$ were provided by Conexão Sistemas de Prótese (Arujá, SP, Brazil). Samples were submitted to surface treatment and divided into four groups:

Porous: samples treated in acidic solutions containing $\mathrm{HNO}_{3}, \mathrm{H}_{2} \mathrm{SO}_{4}$, and $\mathrm{HCl}$ (surface treatment similar to Porous implants available from Conexão Sistemas de Prótese);

PorousNano: treatment similar to Group 1 followed by fluoride ion modification by immersion for one hour in a solution containing fluorine ions;

Porous-FN: treatment similar to Group 1 with FN incorporation;

PorousNano-FN: treatment similar to Group 2 with FN incorporation.

After treatments, samples from the Porous and PorousNano groups were washed with distilled water and absolute alcohol, dried in oven at $70^{\circ} \mathrm{C}$ for two hours, and packed and sterilized by gamma irradiation ( $25 \mathrm{kGy})$.

2.2. Surface Characterization. To characterize the surface morphology and identify differences in samples submitted to treatments with acids and/or fluorides, the samples were characterized by a high-resolution scanning electron microscopy (FEG/EDS, Philips XL30FEG). The results were complemented by analysis with an MFP-3D atomic force microscope (Asylum Research, CA, USA) operating in contact at room temperature mode. The cantilevers used were V shaped, NP-S model (Veeco Probes, CA, USA) with an $0.08 \mathrm{~N} / \mathrm{m}$ spring constant, and calibrated using the thermal noise method. To reduce damage to samples and reduce noise, images were acquired using low-frequency scanning $(1.0 \mathrm{~Hz})$ with $256 \times 256$ pixel resolution. Image processing was performed in the program IGOR PRO (WaveMetrics, Portland, OR, USA) using a MFP-3D platform developed by Asylum Research.

2.3. Identification of Crystalline Phases. An X-ray diffractometer was used to identify crystalline phases on discs. $\mathrm{X}$-ray diffraction for the analysis of thin films (grazing incidence technique) was conducted at $40 \mathrm{kV}$ and $30 \mathrm{~mA}$. A copper anode was used $(\mathrm{Cu}-\mathrm{K} \alpha=1,542 \AA)$ with an $\mathrm{RU}$ 200B model Rigaku generator and $0.02^{\circ}$ step/minute.

2.4. Fibronectin Incorporation. Human serum fibronectin (Sigma-Aldrich Co., São Paulo, Brazil)) was diluted to 
$10 \mathrm{~g} / \mathrm{mL}, \mathrm{pH} 4.5$ in previously filtered $20 \mathrm{mM}$ sodium acetate (Reagen Laboratory Products, Paraná, Brazil) buffer solution. $\mathrm{NaCl}$ was added to the solution to maintain the medium's ionic strength between 0.145 and $0.150 \mathrm{~mol} \cdot \mathrm{dm}^{-3}$.

Samples from the Porous and PorousNano groups were coated with fibronectin at room temperature for 2 hours. Substrates with FN were washed with PBS (phosphate $[0.01 \mathrm{M}]$ buffered saline $[0.15 \mathrm{M}], \mathrm{pH} 7.2)$ to remove nonadsorbed molecules. Then, the adsorbed molecules were detached using $0.1 \%$ trypsin and PBS. One to two minutes later, the excess was removed, and the resulting solution was collected and analyzed with a Spectrum 22PC spectrophotometer to quantify the adsorbed molecules. Spectrophotometry was also used to determine the FN's absorbance on both surfaces (protein concentration in solutions that absorb radiation). Negative (PBS) and positive (FN suspension $100 \mu \mathrm{g} / \mathrm{mL}$ ) controls were performed. The wavelength used was $550 \mathrm{~nm}$ (protein reading).

2.5. Culture of Osteoblasts. Cells were maintained in polystyrene bottles containing DMEM (Dulbecco's Modified Eagle Medium) culture medium with low glucose, 10\% fetal bovine serum (Soromed Industry, São Paulo, Brazil), and $1 \%$ essential amino acids solution (Minimum Essential amino acid solution 100x, Sigma-Aldrich) ascorbic acid $\left(0.15 \mathrm{gL}^{-1}\right.$, Sigma-Aldrich) buffered with $10 \mathrm{mM}$ HEPES (Sigma-Aldrich) and $14.3 \mathrm{mM} \mathrm{NaHCO}_{3}$ (Reagen). The $\mathrm{pH}$ of the medium was adjusted to 7.2. Cultures were incubated at $37^{\circ} \mathrm{C}$ in $5 \% \mathrm{CO}_{2}$ atmosphere. The enzymatic cell detachment technique was used to transpose cells from the stock culture flask to substrates for the adhesion assay. Confluent cultures were treated with $0.2 \%$ trypsin (Difco Microbiology Co., USA) and $0.02 \%$ EDTA (Sigma-Aldrich) in saline solution $(0.8 \% \mathrm{NaCl}$ [Reagan], $0.01 \% \mathrm{KCl}$ [Sigma-Aldrich]; 0.29\% $\mathrm{NaHPO}_{4} \cdot 7 \mathrm{H}_{2} \mathrm{O}$ [Reagan], and $0.02 \% \mathrm{KH}_{2} \mathrm{PO}_{4}$ [SigmaAldrich] in $\mathrm{H}_{2} \mathrm{O}$ ) for 5 minutes at $37^{\circ} \mathrm{C}$. Then, the detached cells were collected, and the proteolytic action of trypsin was inhibited by adding fetal calf serum to the solution. The suspension was then centrifuged at $1500 \mathrm{rpm}$ at $22^{\circ} \mathrm{C}$, and the pelleted cells were resuspended in culture medium without fetal calf serum. The cell concentration/density of the suspension was estimated by counting in a hematimetric Neubauer chamber.

2.6. Interaction of Cells with Samples. After the cell concentration of the suspension was measured in a hematimetric chamber, $10^{6}$ cells $/ \mathrm{mL}$ were taken and allowed to interact with the samples with and without FN coating, which totaled four groups. After an hour of interaction, the supernatants were discarded, and the cells that were attached (adsorbed and adhered) to surfaces were washed with PBS and fixed using glutaraldehyde (2.5\% in PBS). Glutaraldehyde was used as fixative to avoid damaging cell integrity (glutaraldehyde contains two functional groups that link two proteins). This procedure was adopted because the use of formaldehyde (which has only one functional group) as a fixative profoundly deformed the cells. After fixation, cells were trypsinized and counted in a hematimetric Neubauer chamber.
2.7. Surface Radioactivity. Human osteoblastic cells (HOBs) were cultivated to evaluate cell adhesion and proliferation by liquid scintillation counting. Cells from confluent $\mathrm{HOB}$ cultures were detached with trypsin, washed, and counted in a hematimetric chamber. Then, the culture was resuspended in DMEM containing serum and $\left[{ }^{3} \mathrm{H}\right]$-thymidine $(1143 \mathrm{cpm})$. After allowing incorporation for a period of 12 hours, the confluent cells were again detached and washed in DMEM without serum, and a liquid scintillator (Beckman, Rack III) was used to evaluate the radioactivity associated with cells. The resulting values were expressed as counts per minute $(\mathrm{cpm})$. These cells, incorporating $\left[{ }^{3} \mathrm{H}\right]$-thymidine, were associated with different surfaces (Porous, Porous-FN, PorousNano, and PorousNano-FN) for a period of 3 hours, and counts were carried out after 1, 2, and 3 hours. This cell behavior evaluation method allows accurate reproduction, favoring the future applicability of FN incorporation onto surfaces of dental implants.

\section{Results}

3.1. Surface Morphology. Figure 1 shows Porous and PorousNano titanium surfaces before coating with fibronectin. These surfaces exhibited microcavities with different sizes and sharp edges. Immersion into a solution containing fluoride ions (PorousNano) did not change the microcavity morphology, and the sharp edges persisted. A minor modification caused by immersion is shown in Figure 1(c); some white regions are observed when compared with Figure 1(b). At high magnification (Figure 1(d)), the PorousNano group showed evidence of particle clusters at the surface due to immersion in the solution containing fluoride ions. This is the major ultrastructural characteristic of the PorousNano sample.

Figure 2 shows images obtained by atomic force microscopy. In Figure 2(a), the microcavity edges are more flattened but maintain the sharp features that seem to assist or facilitate the adsorption of fibronectin and cells. Figure 2(b) shows the PorousNano sample surface at high magnification, demonstrating that the roughness pattern at the microcavity edges is flattened by immersion treatment in a solution containing fluoride ions.

The surface roughness of the Porous sample (Figure 2(a)) was $1759.7 \mathrm{~nm}( \pm 204.4 \mathrm{~nm})$, whereas the roughness of the PorousNano surface sample (Figure 2(b)) was $1406.5 \mathrm{~nm}$ $( \pm 226.9 \mathrm{~nm})$.

3.2. Identification of Crystalline Phases. Figure 3 shows the $\mathrm{X}$-ray diffraction spectra of the Porous and PorousNano surfaces. Both contain only titanium as the crystalline phase.

3.3. Incorporation of Fibronectin. Porous and PorousNano titanium surfaces were treated with crystal violet $(1 \%$ in PBS), and the stain associated with the surfaces was eluted with methanol. Negative (buffer solution) and positive (FN suspension) controls were assessed by spectrophotometry. The absorbance was proportional to the amount of the cells such that more the cells on the surface corresponded to larger absorbance values. Cells treated with PBS measured at 0.326 


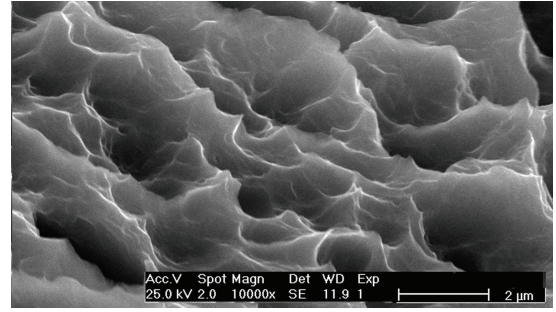

(a)

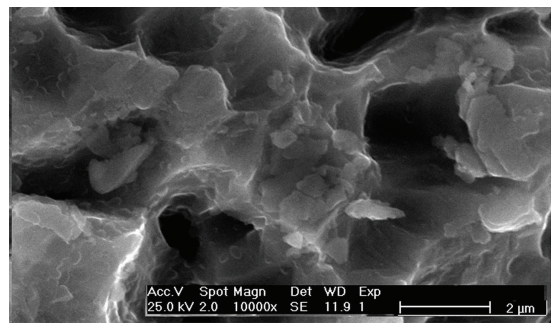

(c)

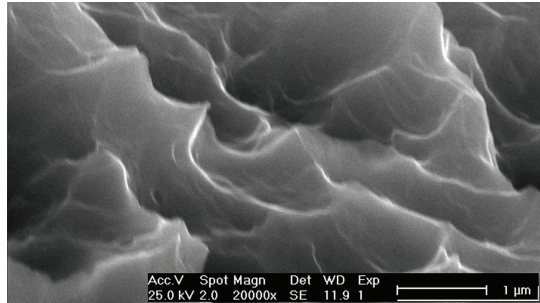

(b)

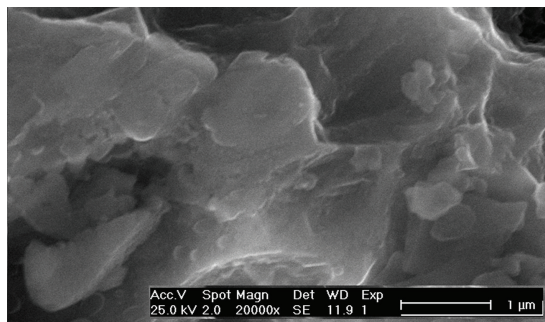

(d)

Figure 1: SEM images of the samples before coating with fibronectin. (a) and (b) Porous samples (acid treatment). (c) and (d) PorousNano samples (acid treatment followed by fluoride ion modification).

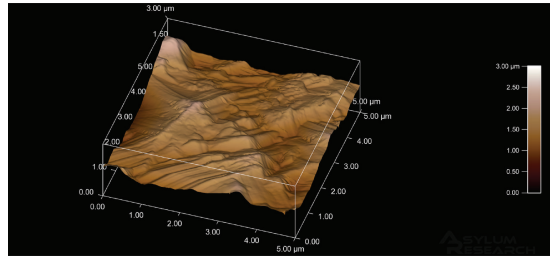

(a)

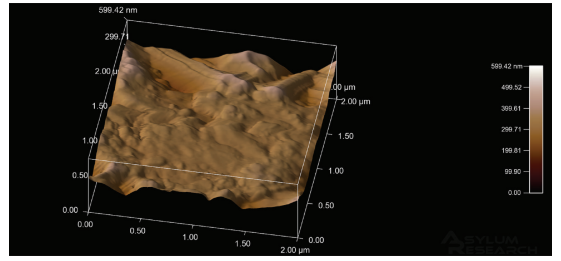

(b)

Figure 2: AFM images: (a) Porous and (b) PorousNano.

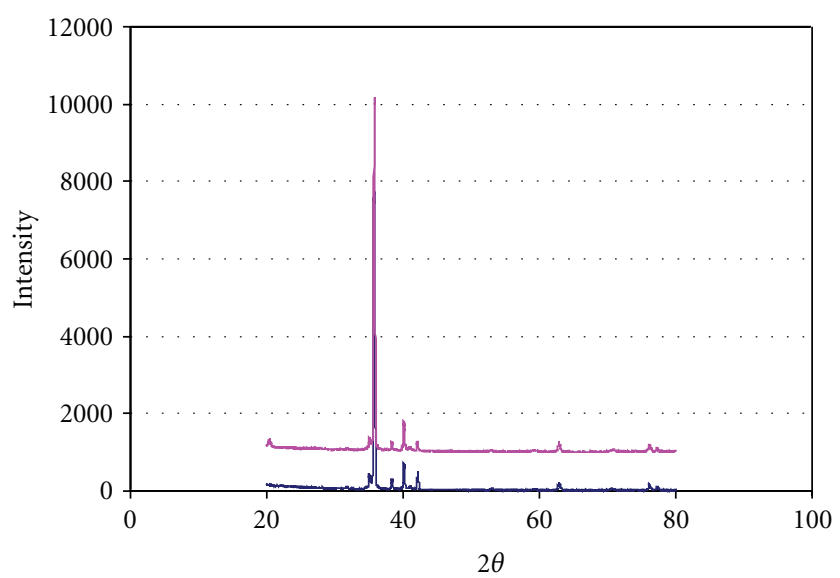

Figure 3: X-ray diffraction spectra of Porous and PorousNano surfaces.

absorbance units (AU) at $550 \mathrm{~nm}$ (reading for proteins). The FN suspension $(100 \mu \mathrm{g} / \mathrm{mL})$ measured at 2.992 units. After the FN incorporation in Porous, and PorousNano tablets, both spectrophotometric measurements were 2.473 absorbance units $(82.6 \%)$ at $550 \mathrm{~nm}$, indicating that the two surfaces exhibit similar behavior with respect to fibronectin incorporation.

3.4. Interaction of Cells with Surfaces. A total of $10^{6}$ human osteoblastic cells/mL were delivered to Porous and PorousNano surfaces, and, after a 1.0 hour interaction, $7.9 \times$ $10^{4}$ cells $/ \mathrm{mL}$ and $2.3 \times 10^{5}$ cells $/ \mathrm{mL}$ were associated with the Porous and PorousNano (no protein coating) surfaces, respectively. The combination of cells to both surfaces, with and without the fibronectin incorporation, resulted in different association indices. For comparison, association index values were considered null for samples without FN. After one hour, the association indices values of cells with samples with FN showed increase of $44.7 \%( \pm 0.8 \%)$ and $57.4 \%( \pm 0.3 \%)$ for Porous-FN and PorousNano-FN surfaces, respectively, compared to the same surfaces without FN.

The cell-surface interaction index of the PorousNano-FN was approximately $28 \%$ higher than that of the Porous-FN.

3.5. Surface Radioactivity. After the incorporation of $\left[{ }^{3} \mathrm{H}\right]$ thymidine for 12 hours, the radioactivity associated with osteoblast cells was evaluated. Subsequently, $1.8 \times$ $10^{6}$ cells $/ \mathrm{mL}$, corresponding to $1,100 \mathrm{cpm}$, were delivered 


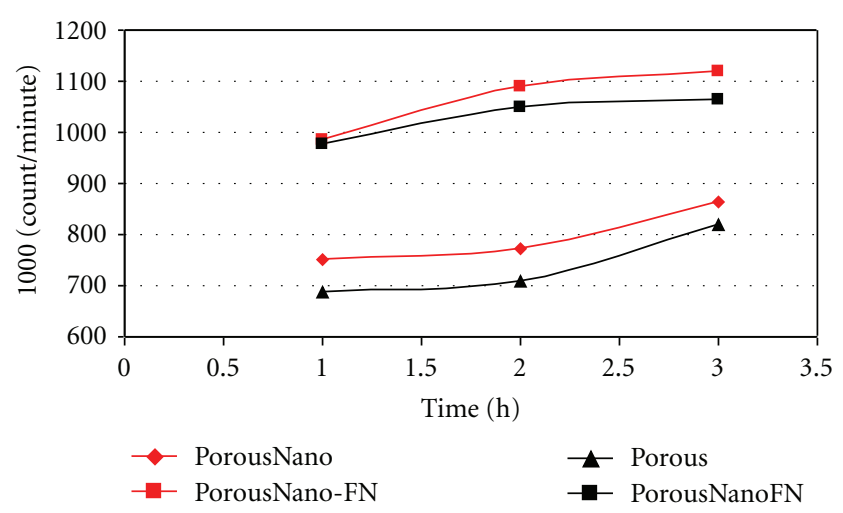

Figure 4: Radioactivity associated with osteoblasts on Porous, Porous-FN, PorousNano, and PorousNano-FN surfaces. The resulting values were expressed as counts per minute (cpm).

to Porous, Porous-FN, PorousNano, and PorousNano-FN surfaces. The results are shown in Figure 4.

After one hour of interaction, $70 \%$ of cells $(0.751 \mathrm{cpm})$ were associated with the PorousNano surface. This number is most likely low because some cells died or were not associated with the sample at the beginning of the process. The number of associated cells increased with interaction time, reaching $0.864 \mathrm{cpm}$ after three hours; that is, there was a $15 \%$ increase in the amount of associated cells due to proliferation and cell division.

Only $64 \%$ of the cells interacting with the Porous surface $(0.687 \mathrm{cpm})$ remained associated after one hour, but this number increased approximately $32 \%$ after 3 hours of interaction, reaching $0.905 \mathrm{cpm}$.

On the Porous-FN surface, $90 \%$ of cells $(0.976 \mathrm{cpm})$ were associated after 1 hour of interaction. The number of attached cells increased 9\% after three hours, reaching $1.064 \mathrm{cpm}$. For the PorousNano-FN surface, 92\% of cells $(0.986 \mathrm{cpm})$ were associated after one hour of interaction, and this number increased by $11.5 \%$ over three hours, reaching $1.100 \mathrm{cpm}$.

\section{Discussion}

Figure 1(a) shows the surface morphology of a Porous sample obtained by immersion treatment in acid solution. The acid etching produces a homogeneous surface characterized by microcavities surrounded by tapered summits. This pattern of roughness produces a homogeneous surface without preferential roughness orientation.

Figure 1(c) shows that the immersion of the Porous surface in a solution containing fluoride ions did not change the microcavity morphology, and the sharp edges persisted. At higher magnification, the presence of flatter areas and smaller micropeaks may be noted although these surfaces remain tapered. This change may be associated with the high reactivity of fluorine ions and the chemical susceptibility of titanium oxide to these ions, which may produce a coalescence of peaks. These results are consistent with those of Ellingsen and Lyngstadaas [9] and Johansson et al. [8], which showed that titanium surfaces treated with fluoride present smoother microtopographies and lower $R_{a}$ values than acidtreated surfaces without fluoride. Figure 1(d) demonstrates the presence of microcavities, summits, and conglomerates on their edges, most likely due to the corrosion process and consequent decrease in surface roughness for the surface subjected to immersion in solution containing fluoride.

Images obtained by atomic force microscopy (Figure 2) show that both the Porous and PorousNano surfaces exhibit microcavities surrounded by summits. Like the highresolution SEM images, the AFM images indicate that summits and microcavities of the PorousNano sample surface have smoother edges although they remain tapered. These sharp edges seem to assist or facilitate the adsorption of FN and cells.

As measured based on the images obtained through AFM, the roughness of the PorousNano surface sample was lower than that of the Porous surface, demonstrating that treatment with fluoride reduced the summit height, most likely due to the reaction of titanium oxide with fluoride ions. This ultrastructural aspect of the summits contributes to the more homogeneous roughness pattern of the PorousNano surface, in addition to the presence of smoother areas and larger microcavities.

The presence of only one crystalline phase of titanium was revealed by X-ray diffraction of the Porous and PorousNano samples. It is likely that the immersion in a solution containing fluoride ions adds only a small amount of this element to the titanium surface and that this trace amount of fluoride cannot be detected by the XRD technique for the analysis of thin films (grazing incidence technique).

Approximately $80 \%$ of the FN allowed to interact with Porous and PorousNano surfaces was adsorbed (2.473 AU). This result demonstrates that the chemical treatment with acids (Porous) and chemical treatment with acids followed by immersion in solution containing fluoride ions (PorousNano) did not affect the incorporation of biomolecule; that is, the presence of the fluoride ion did not influence the protein adsorption. Dos Santos et al. [15] observed that FN adsorption to anodized titanium samples was $68 \%$. It can be concluded that titanium surfaces have an affinity for fibronectin and that differences in the percentage of incorporation in different studies most likely are due to the conditions under which the FN was reacted with the surfaces ( $\mathrm{pH}$ used, for example) and/or the various treatments performed on them.

Cell counting in a hematimetric chamber is a sensitive and accurate technique for the evaluation of cell adhesion to titanium surfaces. In this study, the PorousNano surface showed a stronger association with osteoblastic cells $(2,3 \times$ $10^{5}$ cells $\left./ \mathrm{mL}\right)$ than the Porous surface $\left(7,9 \times 10^{4}\right.$ cells $\left./ \mathrm{mL}\right)$ after one hour of interaction. Because $10^{6}$ cells $/ \mathrm{mL}$ were taken to interact with surfaces, approximately $8 \%$ adhered to the Porous sample, while $23 \%$ were associated with the PorousNano sample. These indices suggest that the surface subjected to chemical treatment followed by immersion in a solution containing fluoride ions favors the adhesion of most cells during the initial interaction period. As mentioned earlier, the association indices of the Porous and PorousNano 
surfaces without fibronectin were considered null for evaluations of the influence of protein on cell behavior. Thus, the number of cells associated with the PorousNano with FN surface increased $57.4 \%$ compared with the same surface without the biological variable. For the Porous with FN surface, the increase in cell adhesion was $44.7 \%$ compared to the same area without the protein. These indices show that the protein variable is responsible for the significant increase in the number of cells attached to the surfaces, confirming the results of $\mathrm{Ku}$ et al. [16], who also reported an increase in the adhesion rate of cells to surfaces treated with recombinant fibronectin. They showed that, for $\mathrm{TiO}_{2}$, cell adhesion was initiated after 3 hours and had significantly lower cell numbers for all measurement points compared with FN. The present work showed the same results.

The cell-surface interaction index of PorousNano with FN was approximately $28 \%$ higher than that of the Porous with FN surface. This study demonstrates that, among the four types of surfaces examined, the PorousNano with fibronectin coating most favors the adsorption and adhesion of osteoblastic cells during the tested interaction period. In addition, the study provides strong evidence that FN incorporation into titanium surfaces is much more relevant for biocompatibility and the consequent acceleration of the osseointegration process than surface treatment with acid and/or immersion in solution containing fluoride ions.

A total of $1.8 \times 10^{6}$ cells $/ \mathrm{mL}(1.100 \mathrm{cpm})$ were allowed to interact with Porous, Porous-FN, PorousNano, and PorousNano-FN titanium surfaces for three hours. After one hour of interaction, $92 \%$ of the cells were associated with the PorousNano-FN surface, and $90 \%$ of cells were associated with the Porous-FN surface, while $70 \%$ and $64 \%$ of cells were associated with the PorousNano and Porous surfaces (without FN), respectively. These results confirm that the protein coating accelerated the adsorption of cells during the initial interaction period (adaptation period). This can be explained by the fact that when fibronectin is allowed to interact with titanium samples under ideal conditions of $\mathrm{pH}$ such that its cryptic sites are exposed, the fibronectin signals to osteoblasts to activate the cell cycle and initiate the secretion of ECM proteins.

The Porous-FN and PorousNano-FN surfaces showed similar behavior during the three-hour interaction, both during the initial adherence of cells (approximately $90 \%$ for both surfaces) and in their proliferation. The cell number increased by $14 \%$ for the sample PorousNano-FN and $12 \%$ for Porous-FN in the first 3 hours of interaction (Figure 4). $\mathrm{Ku}$ et al. [16] also demonstrated that the biomimetization of titanium surfaces with fibronectin increased the adhesion, proliferation, and differentiation rates of cells.

In samples without FN, this study showed that within one-to-three hours of interaction, the number of cells attached to the PorousNano surface increased by $32 \%$, while the number of cells attached to the Porous surface increased by $15 \%$. This difference shows that the surface that received acid treatment followed by immersion in a solution containing fluoride ions (Nano) showed accelerated cell division and proliferation compared to the Porous surface. Figure 4 shows that the PorousNano surface without fibronectin coating exhibited the greatest increase in cpm as a function of time over 3 hours. Ellingsen and Lyngstadaas [9] and Johansson et al. [8] showed that fluoride-treated surfaces have a greater capacity to react with biological tissues and nuclear phosphate crystals in vitro, in addition to offering greater osseointegration resistance in vivo. Although previous studies have used different methodologies for fluoride treatment, their results also suggest that the presence of fluoride ions on titanium surfaces facilitates various osseointegration processes. Analysis of the experimental cell adhesion and proliferation data presented in Figure 4 showed that the cell behavior was similar in all samples containing fibronectin. The results of this study show that the FN is critical to the biocompatibility of surfaces of titanium implants, but when this protein is not present, treatment with acids and fluorides seems to favor more tissue integration than treatment with acid only (i.e., no fluoride).

\section{Conclusions}

Based on the experimental results, it can be concluded that

(a) the surfaces of titanium samples treated with fluoride ions (PorousNano) retained the basic microstructural characteristics of surfaces not treated with fluoride (Porous),

(b) the Porous and PorousNano surfaces incorporated similar levels of FN (approximately 80\%) over the time tested ( 3 hours), demonstrating that the presence of fluoride ions did not influence protein adsorption,

(c) the association indices of $\mathrm{HOB}$ cells to the four tested surfaces suggest that FN incorporation is critical for the in vitro cytocompatibility of surfaces,

(d) FN-treated samples showed significantly higher percentages of associated cells during the initial period of one hour, confirming that FN (the biological variable) had a greater effect on the adhesion and proliferation of cells than the fluoride treatment of titanium surfaces used in this study.

\section{Acknowledgments}

The authors would like to thank the $\mathrm{CNPq}$ for financial support under project 306343/2006-1 and FAPERJ project E26/102.714/2008. They also thank Conexão Sistemas e Prótese for supplying the titanium dental implants and disks.

\section{References}

[1] P.-I. Branemark, "Introduction to osseointegration," in TissueIntegrated Prostheses, Osseointegration in Clinical Dentistry, pp. 11-76, Quintessence, Chicago, Ill, USA, 5th edition, 1985.

[2] C. N. Elias and L. Meirelles, "Improving osseointegration of dental implants," Expert Review of Medical Devices, vol. 7, no. 2, pp. 241-256, 2010.

[3] L. M. Gil, T. C. Ladeira, G. C. Menezes, and F. C. Silva-Filho, "The cell-extracellular matrix-biomaterial interface and the 
biocompatibility of titanium implants," Innovations Implant Journal, vol. 4, pp. 58-64, 2009.

[4] L. Scheideler, F. Rupp, H. P. Wendel, S. Sathe, and J. Geis-Gerstorfer, "Photocoupling of fibronectin to titanium surfaces influences keratinocyte adhesion, pellicle formation and thrombogenicity," Dental Materials, vol. 23, no. 4, pp. 469-478, 2007.

[5] S. A. Cho and K. T. Park, "The removal torque of titanium screw inserted in rabbit tibia treated by dual acid etching," Biomaterials, vol. 24, no. 20, pp. 3611-3617, 2003.

[6] A. Wennerberg, T. Albrektsson, and J. Lindhe, "Surface topography of titanium implants," in Clinical Periodontology and Implant Dentistry, pp. 821-825, 4th edition, 2003.

[7] J. E. Davies, "Understanding peri-implant endosseous healing," Journal of Dental Education, vol. 67, no. 8, pp. 932-949, 2003.

[8] C. B. Johansson, A. Wennerberg, A. Holmen, and J. E. Ellingsen, "Enhanced fixation of bone to fluoride-modified implants," in Proceedings of the 6th World Biomaterials Congress, Sydney, Australia, 2000.

[9] J. E. Ellingsen and S. P. Lyngstadaas, "Increasing biocompatibility by chemical modification of titanium surfaces," in BioImplant Interface: Improving Biomaterials and Tissue Reactions, pp. 323-340, CRC Press, Boca Raton, Fla, USA, 1st edition, 2003.

[10] G. D. Menezes, A Interação de Osteoblastos Humanos com Filmes de Fibronectina Plasmática Humana Constituídos Sob Diferentes Condições de pH [Dissertação (Mestrado)], Universidade Federal do Rio de Janeiro, IBCCF, 2003.

[11] J. M. Park, J. Y. Koak, J. H. Jang, C. H. Han, S. K. Kim, and S. J. Heo, "Osseointegration of anodized titanium implants coated with fibroblast growth factor-fibronectin (FGF-FN) fusion protein," International Journal of Oral and Maxillofacial Implants, vol. 21, no. 6, pp. 859-866, 2006.

[12] M. Antia, G. Baneyx, K. E. Kubow, and V. Vogel, "Fibronectin in aging extracellular matrix fibrils is progressively unfolded by cells and elicits an enhanced rigidity response," Faraday Discussions, vol. 139, pp. 229-249, 2008.

[13] S. Miyamoto, B. Z. Katz, R. M. Lafrenie, and K. M. Yamada, "Fibronectin and integrins in cell adhesion, signaling, and morphogenesis," Annals of the New York Academy of Sciences, vol. 857, pp. 119-129, 1998.

[14] T. A. Petrie, J. E. Raynor, C. D. Reyes, K. L. Burns, D. M. Collard, and A. J. García, "The effect of integrin-specific bioactive coatings on tissue healing and implant osseointegration," Biomaterials, vol. 29, no. 19, pp. 2849-2857, 2008.

[15] A. R. Dos Santos, F. Costa E Silva, and G. A. De Soares, "The interaction between human osteoblastic cells and titanium anodized with sulphuric acid coated or not with human plasma fibronectin," Key Engineering Materials, vol. 396-398, pp. 389-392, 2009.

[16] Y. Ku, C. P. Chung, and J. H. Jang, "The effect of the surface modification of titanium using a recombinant fragment of fibronectin and vitronectin on cell behavior," Biomaterials, vol. 26, no. 25, pp. 5153-5157, 2005. 

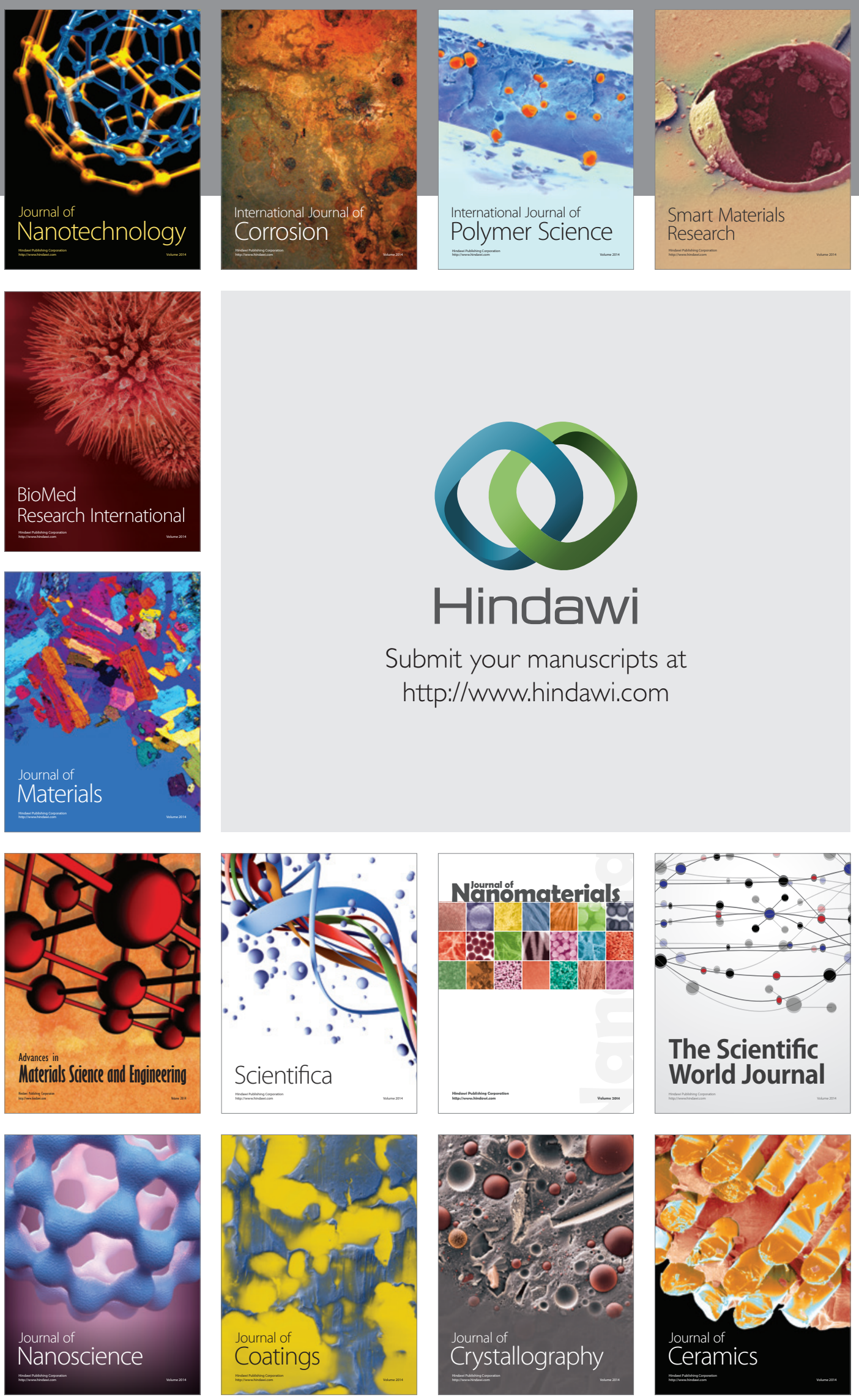

The Scientific World Journal

Submit your manuscripts at

http://www.hindawi.com

\section{World Journal}

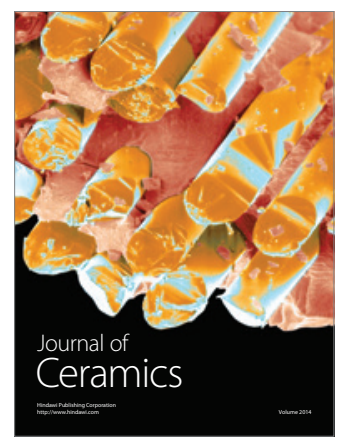

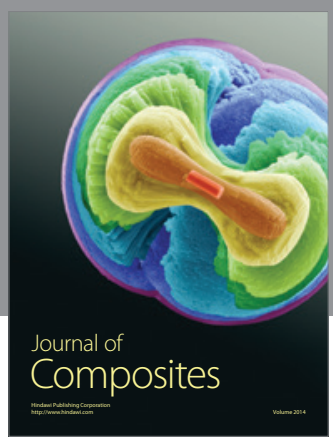
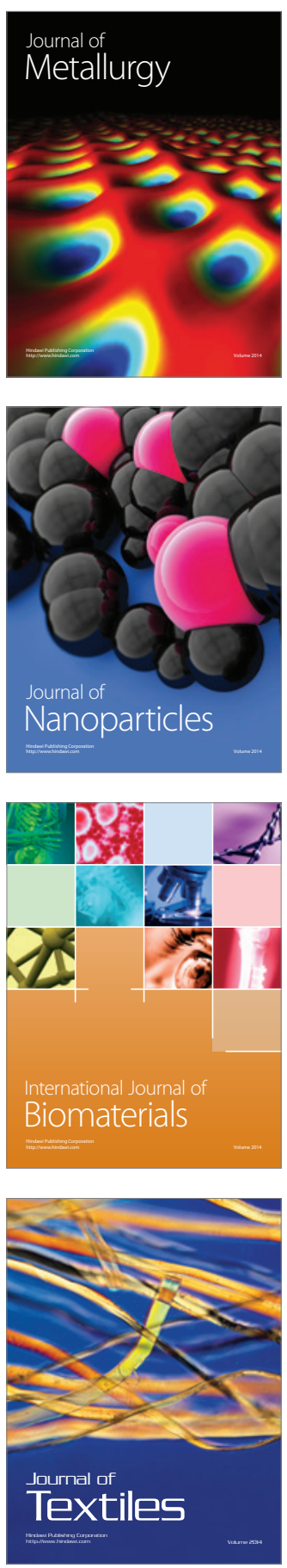